\title{
Upper and Lower Solution Method for Fractional Boundary Value Problems on the Half-Line
}

\author{
Dandan Yang and Chuanzhi Bai \\ Department of Mathematics, Huaiyin Normal University, Huaian, Jiangsu 223300, China \\ Correspondence should be addressed to Chuanzhi Bai; czbai8@sohu.com
}

Received 14 August 2013; Revised 25 October 2013; Accepted 27 October 2013

Academic Editor: Saeid Abbasbandy

Copyright (C) 2013 D. Yang and C. Bai. This is an open access article distributed under the Creative Commons Attribution License, which permits unrestricted use, distribution, and reproduction in any medium, provided the original work is properly cited.

We establish the existence of unbounded solutions for nonlinear fractional boundary value problems on the half-line. By the upper and lower solution method technique, sufficient conditions for the existence of solutions for the fractional boundary value problems are established. An example is presented to illustrate our main result.

\section{Introduction}

Boundary value problems on the half-line arise in the study of radially symmetric solutions of nonlinear elliptic equations and various physical phenomena, such as the theory of drain flows and plasma physics; see [1-10] and the references therein. In 2006, Lian and Ge in [11] investigated the following boundary value problem on the half-line for the second-order differential equation:

$$
\begin{gathered}
x^{\prime \prime}(t)+f\left(t, x(t), x^{\prime}(t)\right)=0, \quad t \in(0,+\infty), \\
x(0)=\alpha x(\eta), \quad \lim _{t \rightarrow \infty} x^{\prime}(t)=0,
\end{gathered}
$$

where $\alpha \in \mathbb{R}, \alpha \neq 1$, and $\eta \in(0, \infty)$ are given. Based on Leray-Schauder continuation theorem, some suitable conditions for the existence of solutions to (1) are established.

On the other hand, fractional calculus is a generalization of the ordinary differentiation and integration to arbitrary noninteger order. Fractional calculus is a wonderful technique to understand memory and hereditary properties of materials and processes. Some recent contributions to fractional differential equations are present in the monographs [12-19]. Very recently, Chen and Tang in [20] considered the following fractional differential boundary value problem on the half-line:

$$
\begin{gathered}
D_{0_{+}}^{\alpha} u(t)=f(t, u(t)), \quad t \in[0, \infty), \\
u(0)=u^{\prime}(0)=u^{\prime \prime}(0)=0, \quad D_{0_{+}}^{\alpha-1} u(t)=\lim _{t \rightarrow \infty} D_{0_{+}}^{\alpha-1} u(t),
\end{gathered}
$$

where $3<\alpha<4$ and $D_{0}^{\alpha}$ is the standard Riemann-Liouville fractional derivative. By the recent Leggett-Williams normtype theorem, the existence of positive solutions is obtained. In 2011, [21] set up the global existence results of solutions of initial value problems on the half-axis as follows:

$$
\begin{gathered}
D_{0_{+}}^{\alpha} x(t)=f(t, x(t)), \quad t \in(0, \infty), 0<\alpha \leq 1, \\
\lim _{t \rightarrow \infty} t^{1-\alpha} x(t)=u_{0},
\end{gathered}
$$

where $D_{0_{+}}^{\alpha}$ is the standard Riemann-Liouville fractional derivative. By constructing a special Banach space and employing fixed point theorems, some sufficient conditions for the existence of solutions are obtained. In [22], the authors studied the following boundary value problem of fractional order on the half-line:

$$
\begin{gathered}
D_{0_{+}}^{\alpha} u(t)+a(t) f\left(t, u(t), D_{0_{+}}^{\alpha-1} u(t)\right)=0, \quad t \in[0, \infty), \\
u(0)=0, \quad \lim _{t \rightarrow \infty} D_{0_{+}}^{\alpha-1} u(t)=u_{\infty},
\end{gathered}
$$


where $1<\alpha \leq 2, f \in C\left([0, \infty) \times \mathbb{R}^{2}, \mathbb{R}\right), D_{0_{+}}^{\alpha-1}$ and $D_{0_{+}}^{\alpha}$ are the standard Riemann-Liouville fractional derivatives. By Schauder's fixed point theorem on an unbounded domain, they obtain the existence result for (4). Some papers have recently been done for fractional boundary value problem on the half-line or unbounded domain, see [22-31].

Inspired by the above-mentioned works, in this paper, we study the existence of solutions to the following fractional differential equations with boundary value problems:

$$
\begin{gathered}
D_{0_{+}}^{\alpha} u(t)+a(t) f\left(t, u(t), D_{0_{+}}^{\alpha-2} u(t), D_{0_{+}}^{\alpha-1} u(t)\right)=0, \\
t \in(0, \infty), \quad 2<\alpha \leq 3, \\
u(0)=D_{0_{+}}^{\alpha-2} u(0)=0, \quad \lim _{t \rightarrow \infty} D_{0_{+}}^{\alpha-1} u(t)=0,
\end{gathered}
$$

where $a(t):[0, \infty) \rightarrow[0, \infty)$, and $D_{0_{+}}^{\alpha}$ is the standard Riemann-Liouville fractional derivative. By upper and lower solution method techniques, the sufficient conditions for solutions to (5) are obtained. Our main findings given in this paper have some new features. Firstly, the like Nagumo condition defined by us plays an important role in the nonlinear term involving the standard Riemann-Liouville derivatives. Secondly, to the best of our knowledge, no work has been done concerning fractional boundary value problems (5) and our method is different from that of [22, $26,31]$. Thirdly, the nonlinear term $f$ may take negative values, and $f(t, x, y, z)$ depends on $z$ allowed to be quadratic, referring to our example. The rest of this paper is organized as follows: in Section 2, we present some preliminaries and some lemmas that will be used in Section 3. The main result and proof will be given in Section 3. In addition, an example is given to demonstrate the application of our main result.

\section{Preliminaries}

We first present some basic definitions and preliminary results about fractional calculus; we refer the reader to $[17,18]$ for more details.

Definition 1 (see [18]). The integral

$$
I_{0_{+}}^{\alpha} f(t)=\frac{1}{\Gamma(\alpha)} \int_{0}^{t} \frac{f(s)}{(t-s)^{1-\alpha}} d s, \quad t>0,
$$

where $\alpha>0$, is called the Riemann-Liouville fractional integral of order $\alpha$ and $\Gamma(\alpha)$ is the Euler gamma function defined by $\Gamma(\alpha)=\int_{0}^{\infty} t^{\alpha-1} e^{-t} d t, \alpha>0$.

Definition 2 (see [17]). A function $f(x)$ given in the interval $[0, \infty)$, the expression

$$
D_{0_{+}}^{\alpha} f(t)=\frac{1}{\Gamma(n-\alpha)}\left(\frac{d}{d t}\right)^{n} \int_{0}^{t} \frac{f(s)}{(t-s)^{\alpha-n+1}} d s,
$$

where $n=[\alpha]+1,[\alpha]$ denotes the integer part of number $\alpha$, is called the Riemann-Liouville fractional derivative of order $\alpha$.
Lemma 3 (see [17]). Let $\alpha>0$ and $u(t) \in C(0, \infty) \bigcap$ $L(0, \infty)$. Then the fractional differential equation

$$
D_{0_{+}}^{\alpha} u(t)=0
$$

has

$$
\begin{array}{r}
u(t)=c_{1} t^{\alpha-1}+c_{2} t^{\alpha-2}+\cdots+c_{n} t^{\alpha-n}, \quad c_{i} \in R, \\
i=0,1,2,3, \ldots, n, \quad n=[\alpha]+1
\end{array}
$$

as a unique solution.

Lemma 4 (see [17]). Assume that $u(t) \in C(0, \infty) \bigcap$ $L(0, \infty)$ with a fractional derivative of order $\alpha>0$ that belongs to $C(0, \infty) \bigcap L(0, \infty)$; then

$$
I_{0_{+}}^{\alpha} D_{0_{+}}^{\alpha} u(t)=u(t)+c_{1} t^{\alpha-1}+c_{2} t^{\alpha-2}+\cdots+c_{n} t^{\alpha-n},
$$

for some $c_{i} \in R, i=0,1,2,3, \ldots, n, n=[\alpha]+1$.

Denote by $C[0, \infty)$ the space of all continuous real functions defined on $[0, \infty)$ and $L_{\text {loc }}(0, \infty)$ the space of all real functions which are Lebesgue integrable on every bounded subinterval of $(0, \infty)$. Denote

$$
\begin{aligned}
E=\{ & x \in C[0, \infty): \sup _{0 \leq t<\infty} \frac{|x(t)|}{(1+t)^{\alpha-1}}<\infty, \\
& \left.\sup _{0 \leq t<\infty}\left|D_{0_{+}}^{\alpha-2} x(t)\right|<\infty, \lim _{t \rightarrow \infty} D_{0_{+}}^{\alpha-1} x(t) \text { exists }\right\}
\end{aligned}
$$

with the norm $\|x\|=\max \left\{\|x\|_{1},\left\|D_{0_{+}}^{\alpha-2} x\right\|_{\infty},\left\|D_{0_{+}}^{\alpha-1} x\right\|_{\infty}\right\}$, where

$$
\begin{gathered}
\|x\|_{1}=\sup _{t \in[0, \infty)}\left|\frac{x(t)}{(1+t)^{\alpha-1}}\right|, \\
\left\|D_{0_{+}}^{\alpha-2} x\right\|_{\infty}=\sup _{t \in[0, \infty)}\left|D_{0_{+}}^{\alpha-2} x(t)\right|, \\
\left\|D_{0_{+}}^{\alpha-1} x\right\|_{\infty}=\sup _{t \in[0, \infty)}\left|D_{0_{+}}^{\alpha-1} x(t)\right| .
\end{gathered}
$$

By standard arguments, it is easy to prove that $(E,\|\cdot\|)$ is a Banach space.

Proposition 5 (see [32]). Assume that $f$ is in $C(0, \infty) \cap$ $L_{\mathrm{loc}}(0, \infty)$ with a fractional derivative of order $0<\alpha<1$ that belongs to $C[0, \infty) \bigcap L_{\mathrm{loc}}[0, \infty)$. Then

$$
I^{\alpha} D^{\alpha} f(x)=f(x)+c x^{\alpha-1},
$$

for some $c \in \mathbb{R}$. When the function $f$ is in $C[0, \infty)$, then $c=0$.

Definition 6. A function $\gamma \in E \bigcap L_{\mathrm{loc}}(0, \infty)$ is called a lower solution of (5) if

$$
\begin{array}{r}
D_{0_{+}}^{\alpha} \gamma(t)+a(t) f\left(t, \gamma(t), D_{0_{+}}^{\alpha-2} \gamma(t), D_{0_{+}}^{\alpha-1} \gamma(t)\right) \geq 0, \\
t \in(0, \infty), \\
\gamma(0) \leq 0, \quad D_{0_{+}}^{\alpha-2} \gamma(0) \leq 0, \quad \lim _{t \rightarrow \infty} D_{0_{+}}^{\alpha-1} \gamma(t) \leq 0 .
\end{array}
$$

Similarly, we can define an upper solution $\beta \in E \bigcap L_{\text {loc }}$ $(0, \infty)$ of $(5)$ by reversing the above inequalities. 
Remark 7. If

$$
D_{0_{+}}^{\alpha-2} \gamma(t) \leq D_{0_{+}}^{\alpha-2} \beta(t), \quad t \in[0, \infty),
$$

and $\gamma(0) \leq 0, \beta(0) \geq 0$, then we have $\gamma(t) \leq \beta(t)$ for all $t \in$ $[0, \infty)$.

We consider the following two cases.

Case 1. Consider $\alpha=3$. The inequality (15) reduces to $\gamma^{\prime}(t) \leq$ $\beta^{\prime}(t)$. Then

$$
\int_{0}^{t} \gamma^{\prime}(s) d s \leq \int_{0}^{t} \beta^{\prime}(s) d s, \quad t \in(0, \infty) ;
$$

that is,

$$
\gamma(t)-\gamma(0) \leq \beta(t)-\beta(0), \quad t \in(0, \infty) .
$$

From the boundary condition $\gamma(0) \leq 0$ and $\beta(0) \geq 0$ we obtain $\gamma(0) \leq \beta(0)$, and

$$
\gamma(t) \leq \beta(t)+\gamma(0)-\beta(0) \leq \beta(t), \quad t \in(0, \infty) .
$$

Thus, $\gamma(t) \leq \beta(t)$ for $t \in[0, \infty)$.

Case 2. Consider $2<\alpha<3$. If (15) holds, then

$$
I_{0_{+}}^{\alpha-2} D_{0_{+}}^{\alpha-2} \gamma(t) \leq I_{0_{+}}^{\alpha-2} D_{0_{+}}^{\alpha-2} \beta(t), \quad t \in[0, \infty) .
$$

Noting that $0<\alpha-2<1$ and $\gamma, \beta \in C[0, \infty)$, from the above inequality and Proposition 5, we have that $\gamma(t) \leq \beta(t)$ for $t \in$ $[0, \infty)$.

For example, consider $\gamma(t)=t^{\alpha-2}, \beta(t)=2 t^{\alpha-2}$. Obviously, $D_{0_{+}}^{\alpha-2} \gamma(t)=\Gamma(\alpha+1), D_{0_{+}}^{\alpha-2} \beta(t)=2 \Gamma(\alpha+1)$. Thus $\gamma(0)=\beta(0)=0$ and

$$
D_{0_{+}}^{\alpha-2} \gamma(t) \leq D_{0_{+}}^{\alpha-2} \beta(t), \quad t \in[0, \infty) .
$$

We also get $\gamma(t) \leq \beta(t)$ for each $t \in[0, \infty)$.

Definition 8. Let $\gamma, \beta \in E \bigcap L_{\text {loc }}(0, \infty)$ be lower and upper solutions to (5) and suppose that (15) holds. A continuous function $f:[0, \infty) \times \mathbb{R}^{3} \rightarrow \mathbb{R}$ is said to satisfy the like Nagumo condition with respect to the pair of functions $\gamma, \beta$, if there exist a nonnegative function $\phi \in C[0, \infty)$ and a positive one $h \in C[0, \infty)$ such that

$$
|f(t, x, y, z)| \leq \phi(t) h(|z|),
$$

for all $0 \leq t<\infty, \gamma(t) \leq x \leq \beta(t), D_{0_{+}}^{\alpha-2} \gamma(t) \leq y \leq$ $D_{0_{+}}^{\alpha-2} \beta(t), z \in \mathbb{R}$, and

$$
\int_{0}^{\infty} \frac{s}{h(s)} d s=\infty
$$

We list some assumptions related to $a(t), \phi(t)$, and $f$ as follows.

$$
\begin{aligned}
& \left(H_{1}\right) \text { Consider } \\
& \qquad \int_{0}^{\infty} \max \{s, 1\} a(s) d s<\infty, \\
& \int_{0}^{\infty} \max \{s, 1\} a(s) \phi(s) d s<\infty .
\end{aligned}
$$

$\left(H_{2}\right)$ Let $f:[0, \infty) \times \mathbb{R}^{3} \rightarrow \mathbb{R}$ be a continuous function satisfying the like Nagumo condition with respect to the pair of functions $\gamma, \beta$, such that

$$
\begin{array}{r}
f(t, \gamma(t), y, z) \leq f(t, x, y, z) \leq f(t, \beta(t), y, z), \\
\quad \text { for }(t, x, y, z) \in[0, \infty) \times[\gamma(t), \beta(t)] \times \mathbb{R}^{2} .
\end{array}
$$

Lemma 9. Let $\sigma \in C(0, \infty) \bigcap L(0, \infty)$, then the fractional boundary value problem

$$
\begin{aligned}
& D_{0_{+}}^{\alpha} u(t)+\sigma(t)=0, \quad t \in(0, \infty), 2<\alpha \leq 3, \\
& u(0)=D_{0_{+}}^{\alpha-2} u(0)=0, \quad \lim _{t \rightarrow \infty} D_{0_{+}}^{\alpha-1} u(t)=0
\end{aligned}
$$

has a unique solution $u(t)=\int_{0}^{\infty} G(t, s) \sigma(s) d s$, where

$$
G(t, s)=\frac{1}{\Gamma(\alpha)} \begin{cases}t^{\alpha-1}-(t-s)^{\alpha-1}, & 0 \leq s \leq t<\infty, \\ t^{\alpha-1}, & 0 \leq t \leq s<\infty .\end{cases}
$$

Proof. By Lemma 4, the solution of (25) has the following form:

$$
\begin{aligned}
u(t)= & -\frac{1}{\Gamma(\alpha)} \int_{0}^{t}(t-s)^{\alpha-1} \sigma(s) d s \\
& +c_{1} t^{\alpha-1}+c_{2} t^{\alpha-2}+c_{3} t^{\alpha-3} .
\end{aligned}
$$

By boundary value condition $u(0)=0$, one has $c_{3}=0$. Thus, we have by (27) that

$$
\begin{aligned}
D_{0_{+}}^{\alpha-2} u(t) & =-D_{0_{+}}^{\alpha-2} I_{0_{+}}^{\alpha} \sigma(t)+c_{1} D_{0_{+}}^{\alpha-2} t^{\alpha-1}+c_{2} D_{0_{+}}^{\alpha-2} t^{\alpha-2} \\
& =-\int_{0}^{t}(t-s) \sigma(s) d s+c_{1} \Gamma(\alpha) t+c_{2} \Gamma(\alpha-1) .
\end{aligned}
$$

Substituting the condition $D_{0_{+}}^{\alpha-2} u(0)=0$ into (28), we obtain $c_{2}=0$. Together with $\lim _{t \rightarrow \infty} D_{0_{+}}^{\alpha-1} u(t)=0$, we get

$$
c_{1}=\frac{1}{\Gamma(\alpha)} \int_{0}^{\infty} \sigma(s) d s
$$

Hence, we have

$$
\begin{aligned}
u(t)= & -\frac{1}{\Gamma(\alpha)} \int_{0}^{t}(t-s)^{\alpha-1} \sigma(s) d s+\frac{t^{\alpha-1}}{\Gamma(\alpha)} \int_{0}^{\infty} \sigma(s) d s \\
= & -\frac{1}{\Gamma(\alpha)} \int_{0}^{t}(t-s)^{\alpha-1} \sigma(s) d s \\
& +\frac{1}{\Gamma(\alpha)} \int_{0}^{t} t^{\alpha-1} \sigma(s) d s+\frac{1}{\Gamma(\alpha)} \int_{t}^{\infty} t^{\alpha-1} \sigma(s) d s \\
= & \frac{1}{\Gamma(\alpha)} \int_{0}^{t}\left[t^{\alpha-1}-(t-s)^{\alpha-1}\right] \sigma(s) d s \\
& +\frac{1}{\Gamma(\alpha)} \int_{t}^{\infty} t^{\alpha-1} \sigma(s) d s \\
= & \int_{0}^{\infty} G(t, s) \sigma(s) d s .
\end{aligned}
$$


Lemma 10. The function $G(t, s)$ defined by (26) satisfies the following properties:

(1) $G(t, s)$ is a continuous function and $G(t, s) \geq 0$ for $[0, \infty) \times[0, \infty)$

(2) $G(t, s) \leq t^{\alpha-1} / \Gamma(\alpha)$, for $(t, s) \in[0, \infty) \times[0, \infty)$.

The proof is easy, so we omit it here.

Set $C_{l}:=\left\{y \in C[0, \infty): \lim _{t \rightarrow \infty} y(t)\right.$ exists $\}$. For $y \in C_{l}$, define $\|y\|:=\sup _{t \in[0, \infty)}|y(t)|$. Then $C_{l}$ is a Banach space.

Lemma 11 (see [33]). Let $M \subset C_{l}$. Then $M$ is relatively compact if the following conditions hold:

(a) $M$ is bounded in $C_{l}$;

(b) the functions belonging to $M$ are locally equicontinuous on $[0, \infty)$;

(c) the functions from $M$ are equiconvergent; that is given $\epsilon>0$, there corresponds $T(\epsilon)>0$ such that $|x(t)-x(\infty)|<\epsilon$, and $x \in M$.

By Lemma 11, similar to the proof of Theorem 2.3 in [34], we can easily have the following lemma.

Lemma 12. Let $M \subset E$. Then $M$ is relatively compact if the following conditions hold:

(1) $M$ is bounded in E;

(2) the functions belonging to $\left\{y: y=x /(1+t)^{\alpha-1}, x \in\right.$ $M\},\left\{z: z=D_{0_{+}}^{\alpha-2} x(t), x \in M\right\}$, and $\{w: w=$ $\left.D_{0_{+}}^{\alpha-1} x(t), x \in M\right\}$, are locally equicontinuous on $[0, \infty)$;

(3) the functions from $\left\{y: y=x /(1+t)^{\alpha-1}, x \in M\right\}$, $\left\{z: z=D_{0_{+}}^{\alpha-2} x(t), x \in M\right\}$, and $\left\{w: w=D_{0_{+}}^{\alpha-1} x(t)\right.$, $x \in M\}$ are equiconvergent at $\infty$.

Define the auxiliary functions

$$
\begin{gathered}
w(t, x)= \begin{cases}\gamma(t), & x<\gamma(t), \\
x(t), & \gamma(t) \leq x \leq \beta(t), \\
\beta(t), & x>\beta(t),\end{cases} \\
w_{1}(t, y)= \begin{cases}D_{0_{+}}^{\alpha-2} \gamma(t), & y<D_{0_{+}}^{\alpha-2} \gamma(t), \\
y(t), & D_{0_{+}}^{\alpha-2} \gamma(t) \leq y \leq D_{0_{+}}^{\alpha-2} \beta(t), \\
D_{0_{+}}^{\alpha-2} \beta(t), & y>D_{0_{+}}^{\alpha-2} \beta(t) .\end{cases}
\end{gathered}
$$

Consider the following boundary value problem:

$$
\begin{array}{r}
D_{0_{+}}^{\alpha} u(t)+a(t) f^{*}\left(t, u(t), D_{0_{+}}^{\alpha-2} u(t), D_{0_{+}}^{\alpha-1} u(t)\right)=0, \\
t \in(0, \infty), \quad 2<\alpha \leq 3, \\
u(0)=D_{0_{+}}^{\alpha-2} u(0)=0, \quad \lim _{t \rightarrow \infty} D_{0_{+}}^{\alpha-1} u(t)=0,
\end{array}
$$

where

$$
\begin{aligned}
f^{*}(t, x, y, z)= & f\left(t, w_{0}(t, x), w_{1}(t, y), z\right) \\
& +\frac{w_{1}(t, y)-y}{1+\left|w_{1}(t, y)-y\right|} .
\end{aligned}
$$

For each $u \in E$, it follows from (21), (23), and (33) that

$$
\begin{aligned}
& \left|\int_{0}^{\infty} a(s) f^{*}\left(s, u(s), D_{0_{+}}^{\alpha-2} u(s), D_{0_{+}}^{\alpha-1} u(s)\right) d s\right| \\
& \quad \leq \int_{0}^{\infty} a(s)\left[\phi(s) h\left(\left|D_{0_{+}}^{\alpha-1} u(s)\right|\right)+1\right] d s \\
& \quad \leq \int_{0}^{\infty} a(s)\left(H_{0} \phi(s)+1\right) d s \\
& \quad \leq \int_{0}^{\infty} \max \{s, 1\} a(s)\left(H_{0} \phi(s)+1\right) d s<\infty,
\end{aligned}
$$

where $H_{0}=\max _{0 \leq t \leq\left\|D_{0_{+}}^{\alpha-1} u\right\|_{\infty}} h(t)$. From (34) and Lemma 9, we know that $\mathrm{u}$ is a solution of (32) if and only if $u$ solves the operator equation $T u=u$, and $T$ is defined by

$$
\begin{array}{r}
T u(t) \\
=\int_{0}^{\infty} G(t, s) a(s) f^{*}\left(s, u(s), D_{0_{+}}^{\alpha-2} u(s), D_{0_{+}}^{\alpha-1} u(s)\right) d s, \\
u \in E, \quad t \in[0, \infty) .
\end{array}
$$

Lemma 13. Suppose that $\left(H_{1}\right)-\left(H_{2}\right)$ hold; then $T: E \rightarrow E$ (the operator defined in (35)) is completely continuous.

Proof. Consider the following.

Step 1. $T: E \rightarrow E$ is well defined. For $u \in E$, it follows from (34) that

$$
\begin{aligned}
& \int_{0}^{\infty} s a(s)\left(H_{0} \phi(s)+1\right) d s \\
& \quad \leq \int_{0}^{\infty} \max \{s, 1\} a(s)\left(H_{0} \phi(s)+1\right) d s<\infty,
\end{aligned}
$$

which implies

$$
\lim _{t \rightarrow \infty} t a(t)\left(H_{0} \phi(t)+1\right)=0 .
$$

We also have

$$
\begin{array}{r}
\int_{t}^{\infty} a(s)\left(H_{0} \phi(s)+1\right) d s \leq \int_{t}^{\infty} s a(s)\left(H_{0} \phi(s)+1\right) d s \\
t \geq 1
\end{array}
$$

Combining (36) with (38), one has

$$
\lim _{t \rightarrow \infty} \int_{t}^{\infty} a(s)\left(H_{0} \phi(s)+1\right) d s=0 .
$$

If we apply Lebesgue dominated convergence theorem with (37) and (39), then

$$
\begin{aligned}
& \lim _{t \rightarrow \infty} \frac{|T u(t)|}{(1+t)^{\alpha-1}} \\
& \quad \leq \lim _{t \rightarrow \infty} \int_{0}^{\infty} \frac{G(t, s)}{(1+t)^{\alpha-1}} a(s)\left(H_{0} \phi(s)+1\right) d s=0,
\end{aligned}
$$


which yields

$$
\lim _{t \rightarrow \infty} \frac{T u(t)}{(1+t)^{\alpha-1}}=0
$$

that is,

$$
\sup _{0 \leq t<\infty} \frac{|T u(t)|}{(1+t)^{\alpha-1}}<\infty .
$$

By virtue of (34), we have

$$
\begin{aligned}
& \sup _{0 \leq t<\infty}\left|D_{0^{+}}^{\alpha-2} T u(t)\right| \\
& =\sup _{0 \leq t<\infty} \mid D_{0^{+}}^{\alpha-2} \int_{t}^{\infty} G(t, s) a(s) f^{*}\left(s, u(s), D_{0^{+}}^{\alpha-2} u(s),\right. \\
& \left.D_{0^{+}}^{\alpha-1} u(s)\right) d s \\
& =\sup _{0 \leq t<\infty} \mid \int_{0}^{t} s a(s) f^{*}\left(s, u(s), D_{0^{+}}^{\alpha-2} u(s), D_{0^{+}}^{\alpha-1} u(s)\right) d s \\
& +\int_{t}^{\infty} \operatorname{ta}(s) f^{*}\left(s, u(s), D_{0^{+}}^{\alpha-2} u(s),\right. \\
& \left.D_{0^{+}}^{\alpha-1} u(s)\right) d s \\
& \leq \sup _{0 \leq t<\infty} \int_{0}^{t} s a(s)\left(H_{0} \phi(s)+1\right) d s \\
& +\int_{t}^{\infty} \operatorname{ta}(s)\left(H_{0} \phi(s)+1\right) d s \\
& \leq \int_{0}^{\infty} s a(s)\left(H_{0} \phi(s)+1\right) d s \\
& \leq \int_{0}^{\infty} \max \{s, 1\} a(s)\left(H_{0} \phi(s)+1\right) d s<\infty .
\end{aligned}
$$

It follows from (39) that

$$
\begin{aligned}
& \left|\int_{t}^{\infty} a(s) f^{*}\left(s, u(s), D_{0_{+}}^{\alpha-2} u(s), D_{0_{+}}^{\alpha-1} u(s)\right) d s\right| \\
& \quad \leq \int_{t}^{\infty} a(s)\left(H_{0} \phi(s)+1\right) d s \longrightarrow 0, \quad t \longrightarrow \infty .
\end{aligned}
$$

Therefore, we have

$$
\begin{aligned}
& \lim _{t \rightarrow \infty} D_{0_{+}}^{\alpha-1} T u(t) \\
& \quad=\lim _{t \rightarrow \infty} \int_{t}^{\infty} a(s) f^{*}\left(s, u(s), D_{0^{+}}^{\alpha-2} u(s), D_{0^{+}}^{\alpha-1} u(s)\right) d s \\
& \quad=0 .
\end{aligned}
$$

Step 2. $T: E \rightarrow E$ is continuous. For any convergent sequence $u_{n} \rightarrow u$ in $E$, we find

$$
\begin{gathered}
u_{n}(t) \longrightarrow u(t), \quad D_{0_{+}}^{\alpha-2} u_{n} \longrightarrow D_{0_{+}}^{\alpha-2} u, \\
D_{0_{+}}^{\alpha-1} u_{n} \longrightarrow D_{0_{+}}^{\alpha-1} u, \quad \text { as } n \longrightarrow \infty, t \in[0, \infty) .
\end{gathered}
$$

From continuity of $f^{*}$, we obtain

$$
\begin{array}{r}
\mid f^{*}\left(s, u_{n}(s), D_{0^{+}}^{\alpha-2} u_{n}(s), D_{0^{+}}^{\alpha-1} u_{n}(s)\right) \\
-f^{*}\left(s, u(s), D_{0^{+}}^{\alpha-2} u(s), D_{0^{+}}^{\alpha-1} u(s)\right) \mid \longrightarrow 0, \\
n \longrightarrow \infty, \quad \text { for any } t \in[0, \infty) .
\end{array}
$$

Since $u_{n} \rightarrow u$, we have $\sup _{n \in N}\left\|D_{0_{+}}^{\alpha-1} u\right\|_{\infty}<\infty$. Set

$$
H_{p}=\max _{0 \leq t \leq \max \left\{\left\|D_{0_{+}}^{\alpha-1} u\right\|_{\infty}, \sup _{n \in N}\left\|D_{0_{+}}^{\alpha-1} u\right\|_{\infty}\right\}} h(t)
$$

which follows that

$$
\begin{gathered}
\int_{0}^{\infty} s a(s) \mid f^{*}\left(s, u_{n}(s), D_{0^{+}}^{\alpha-2} u_{n}(s), D_{0^{+}}^{\alpha-1} u_{n}(s)\right) \\
-f^{*}\left(s, u(s), D_{0^{+}}^{\alpha-2} u(s), D_{0^{+}}^{\alpha-1} u(s)\right) \mid d s \\
\leq 2 \int_{0}^{\infty} s a(s)\left(H_{p} \phi(s)+1\right) d s<\infty .
\end{gathered}
$$

Thus, applying the Lebesgue dominated convergence theorem and then using (49), we have

$$
\begin{aligned}
& \left\|T u_{n}-T u\right\|_{1} \\
& =\sup _{0 \leq t<\infty} \frac{\left|T u_{n}(t)-T u(t)\right|}{(1+t)^{\alpha-1}} \\
& =\sup _{0 \leq t<\infty} \mid \int_{0}^{\infty} \frac{G(t, s)}{(1+t)^{\alpha-1}} a(s) \\
& \quad \times\left(f^{*}\left(s, u_{n}(s), D_{0^{+}}^{\alpha-2} u_{n}(s), D_{0^{+}}^{\alpha-1} u_{n}(s)\right)\right. \\
& \left.\quad-f^{*}\left(s, u(s), D_{0^{+}}^{\alpha-2} u(s), D_{0^{+}}^{\alpha-1} u(s)\right)\right) \mid \\
& \leq \frac{1}{\Gamma(\alpha)} \int_{0}^{\infty} a(s) \mid f^{*}\left(s, u_{n}(s), D_{0^{+}}^{\alpha-2} u_{n}(s), D_{0^{+}}^{\alpha-1} u_{n}(s)\right) \\
& \quad-f^{*}\left(s, u(s), D_{0^{+}}^{\alpha-2} u(s), D_{0^{+}}^{\alpha-1} u(s)\right) \mid
\end{aligned}
$$

$\longrightarrow 0$

Thus, we conclude that $T u \in E$. 
as $n \rightarrow \infty$. On the other hand, we have

$$
\begin{aligned}
& \left\|D_{0_{+}}^{\alpha-2} T u_{n}-D_{0_{+}}^{\alpha-2} T u\right\|_{\infty} \\
& =\sup _{0 \leq t<\infty}\left|D_{0_{+}}^{\alpha-2} T u_{n}(t)-D_{0_{+}}^{\alpha-2} T u(t)\right| \\
& =\sup _{0 \leq t<\infty} \mid \int_{0}^{t} s a(s) \\
& \times\left(f^{*}\left(s, u_{n}(s), D_{0^{+}}^{\alpha-2} u_{n}(s), D_{0^{+}}^{\alpha-1} u_{n}(s)\right)\right. \\
& \left.-f^{*}\left(s, u(s), D_{0^{+}}^{\alpha-2} u(s), D_{0^{+}}^{\alpha-1} u(s)\right)\right) d s \\
& +\int_{t}^{\infty} \operatorname{ta}(s) \\
& \times\left(f^{*}\left(s, u_{n}(s), D_{0^{+}}^{\alpha-2} u_{n}(s), D_{0^{+}}^{\alpha-1} u_{n}(s)\right)\right. \\
& -f^{*}\left(s, u(s), D_{0^{+}}^{\alpha-2} u(s),\right. \\
& \left.\left.D_{0^{+}}^{\alpha-1} u(s)\right)\right) d s \\
& \leq \sup _{0 \leq t<\infty}\left[\int_{0}^{t} s a(s)\right. \\
& \times \mid f^{*}\left(s, u_{n}(s), D_{0^{+}}^{\alpha-2} u_{n}(s), D_{0^{+}}^{\alpha-1} u_{n}(s)\right) d s \\
& -f^{*}\left(s, u(s), D_{0^{+}}^{\alpha-2} u(s), D_{0^{+}}^{\alpha-1} u(s)\right) \mid d s \\
& +\int_{t}^{\infty} s a(s) \\
& \times \mid f^{*}\left(s, u_{n}(s), D_{0^{+}}^{\alpha-2} u_{n}(s), D_{0^{+}}^{\alpha-1} u_{n}(s)\right) \\
& -f^{*}\left(s, u(s), D_{0^{+}}^{\alpha-2} u(s),\right. \\
& \left.\left.D_{0^{+}}^{\alpha-1} u(s)\right) \mid d s\right] \\
& =\int_{0}^{\infty} s a(s) \\
& \times \mid f^{*}\left(s, u_{n}(s), D_{0^{+}}^{\alpha-2} u_{n}(s), D_{0^{+}}^{\alpha-1} u_{n}(s)\right) \\
& -f^{*}\left(s, u(s), D_{0^{+}}^{\alpha-2} u(s), D_{0^{+}}^{\alpha-1} u(s)\right) \mid d s \longrightarrow 0, \\
& n \longrightarrow \infty \text {. }
\end{aligned}
$$

From (49), it is clear that

$$
\begin{aligned}
\int_{0}^{\infty} a(s) \mid f^{*} & \left(s, u_{n}(s), D_{0^{+}}^{\alpha-2} u_{n}(s), D_{0^{+}}^{\alpha-1} u_{n}(s)\right) \\
& -f^{*}\left(s, u(s), D_{0^{+}}^{\alpha-2} u(s), D_{0^{+}}^{\alpha-1} u(s)\right) \mid<+\infty
\end{aligned}
$$

Moreover, by (52), we have

$$
\begin{aligned}
& \left\|D_{0_{+}}^{\alpha-1} T u_{n}-D_{0_{+}}^{\alpha-1} T u\right\|_{\infty} \\
& =\sup _{0 \leq t<\infty}\left|D_{0_{+}}^{\alpha-1} T u_{n}(t)-D_{0_{+}}^{\alpha-1} T u(t)\right| \\
& =\sup _{0 \leq t<\infty} \mid \int_{t}^{\infty} a(s)\left(f^{*}\left(s, u_{n}(s), D_{0^{+}}^{\alpha-2} u_{n}(s), D_{0^{+}}^{\alpha-1} u_{n}(s)\right)\right. \\
& \left.\quad-f^{*}\left(s, u(s), D_{0^{+}}^{\alpha-2} u(s), D_{0^{+}}^{\alpha-1} u(s)\right)\right) \mid \\
& \leq \int_{0}^{\infty} a(s) \mid f^{*}\left(s, u_{n}(s), D_{0^{+}}^{\alpha-2} u_{n}(s), D_{0^{+}}^{\alpha-1} u_{n}(s)\right) \\
& \quad-f^{*}\left(s, u(s), D_{0^{+}}^{\alpha-2} u(s), D_{0^{+}}^{\alpha-1} u(s)\right) \mid \longrightarrow 0, \\
& \quad n \longrightarrow \infty .
\end{aligned}
$$

Therefore, combining (50), (51), and (53), we get $\| T u_{n}-$ $T u \| \rightarrow 0$, as $n \rightarrow \infty$; then we claim that $T: E \rightarrow E$ is continuous.

Step 3. $T: E \rightarrow E$ is compact. Let $A$ be any bounded subset of $E$; then, for $u \in A$, set

$$
H_{q}=\sup _{0 \leq t \leq\left\|D_{0_{+}}^{\alpha-1}\right\|_{\infty}, u \in A} h(t)<\infty,
$$

in a similar manner as (50), (51), and (53); we have by (21) and (23) that

$$
\begin{aligned}
& \|T u\|_{1} \\
& =\sup _{0 \leq t<\infty} \frac{|T u(t)|}{(1+t)^{\alpha-1}} \\
& \leq \sup _{0 \leq t<\infty} \int_{0}^{\infty} \frac{G(t, s)}{(1+t)^{\alpha-1}} a(s) \\
& \quad \times\left|f^{*}\left(s, u(s), D_{0^{+}}^{\alpha-2} u(s), D_{0^{+}}^{\alpha-1} u(s)\right)\right| d s \\
& \leq \frac{1}{\Gamma(\alpha)} \int_{0}^{\infty} a(s) \\
& \quad \times\left|f^{*}\left(s, u(s), D_{0^{+}}^{\alpha-2} u(s), D_{0^{+}}^{\alpha-1} u(s)\right)\right| d s \\
& \qquad D_{0_{+}}^{\alpha-2} T u \|_{\infty} \leq \int_{0}^{\infty} s a(s) \\
& \quad \times\left|f^{*}\left(s, u(s), D_{0^{+}}^{\alpha-2} u(s), D_{0^{+}}^{\alpha-1} u(s)\right)\right| d s \\
& \leq \int_{0}^{\infty} s a(s)\left(H_{q} \phi(s)+1\right) d s<\infty,
\end{aligned}
$$




$$
\begin{aligned}
& \left\|D_{0_{+}}^{\alpha-1} T u\right\|_{\infty} \\
& \quad \leq \int_{0}^{\infty} a(s)\left|f^{*}\left(s, u(s), D_{0^{+}}^{\alpha-2} u(s), D_{0^{+}}^{\alpha-1} u(s)\right)\right| d s \\
& \quad \leq \int_{0}^{\infty} a(s)\left(H_{q} \phi(s)+1\right) d s<\infty,
\end{aligned}
$$

$$
\begin{aligned}
& +t_{1} \int_{t_{1}}^{t_{2}} a(s)\left(H_{q} \phi(s+1)\right) d s \\
& +\left|t_{2}-t_{1}\right| \int_{t_{2}}^{\infty} a(s)\left(H_{q} \phi(s+1)\right) d s,
\end{aligned}
$$

which implies that $\|T u\|<\infty$. Hence, $T A$ is uniformly bounded. Meanwhile, for any $B>0$, for $t_{1}, t_{2} \in[0, B]$, we have

$$
\begin{aligned}
& \left|\frac{T u\left(t_{1}\right)}{\left(1+t_{1}\right)^{\alpha-1}}-\frac{T u\left(t_{2}\right)}{\left(1+t_{2}\right)^{\alpha-1}}\right| \\
& =\mid \int_{0}^{\infty}\left(\frac{G\left(t_{1}, s\right)}{\left(1+t_{1}\right)^{\alpha-1}}-\frac{G\left(t_{2}, s\right)}{\left(1+t_{2}\right)^{\alpha-1}}\right) \\
& \quad \times a(s) f^{*}\left(s, u(s), D_{0_{+}}^{\alpha-2} u(s), D_{0_{+}}^{\alpha-1} u(s)\right) \mid \\
& \leq \int_{0}^{\infty}\left|\frac{G\left(t_{1}, s\right)}{\left(1+t_{1}\right)^{\alpha-1}}-\frac{G\left(t_{2}, s\right)}{\left(1+t_{2}\right)^{\alpha-1}}\right| \\
& \quad \times a(s)\left(H_{q} \phi(s)+1\right) d s \longrightarrow 0, \quad t_{1} \longrightarrow t_{2} .
\end{aligned}
$$

We also have

$$
\begin{aligned}
& \left|D_{0_{+}}^{\alpha-2} T u\left(t_{1}\right)-D_{0_{+}}^{\alpha-2} T u\left(t_{2}\right)\right| \\
& =\mid \int_{0}^{t_{1}} s a(s) f^{*}\left(s, u(s), D_{0_{+}}^{\alpha-2} u(s), D_{0_{+}}^{\alpha-1} u(s)\right) d s \\
& \quad+\int_{t_{1}}^{\infty} t_{1} a(s) f^{*}\left(s, u(s), D_{0_{+}}^{\alpha-2} u(s), D_{0_{+}}^{\alpha-1} u(s)\right) d s \\
& \quad-\int_{0}^{t_{2}} s a(s) f^{*}\left(s, u(s), D_{0_{+}}^{\alpha-2} u(s), D_{0_{+}}^{\alpha-1} u(s)\right) d s \\
& \quad-\int_{t_{2}}^{\infty} t_{2} a(s) f^{*}\left(s, u(s), D_{0_{+}}^{\alpha-2} u(s), D_{0_{+}}^{\alpha-1} u(s)\right) d s \mid \\
& \leq\left|\int_{t_{1}}^{t_{2}} s a(s) f^{*}\left(s, u(s), D_{0_{+}}^{\alpha-2} u(s), D_{0_{+}}^{\alpha-1} u(s)\right) d s\right| \\
& \quad+\left|t_{1} \int_{t_{1}}^{t_{2}} a(s) f^{*}\left(s, u(s), D_{0_{+}}^{\alpha-2} u(s), D_{0_{+}}^{\alpha-1} u(s)\right) d s\right| \\
& \quad+\mid\left(t_{2}-t_{1}\right) \int_{t_{2}}^{\infty} a(s) f^{*}\left(s, u(s), D_{0_{+}}^{\alpha-2} u(s),\right. \\
& \leq \int_{t_{1}}^{t_{2}} s a(s)\left(H_{q} \phi(s+1)\right) d s
\end{aligned}
$$

which approaches 0 , as $t_{1} \rightarrow t_{2}$. Furthermore, we have

$$
\begin{aligned}
& \left|D_{0_{+}}^{\alpha-1} T u\left(t_{1}\right)-D_{0_{+}}^{\alpha-1} T u\left(t_{2}\right)\right| \\
& \quad=\left|\int_{t_{1}}^{t_{2}} a(s) f^{*}\left(s, u(s), D_{0_{+}}^{\alpha-2} u(s), D_{0_{+}}^{\alpha-1} u(s)\right) d s\right| \\
& \quad \leq \int_{t_{1}}^{t_{2}} a(s)\left(H_{q} \phi(s)+1\right) d s,
\end{aligned}
$$

which approaches 0 , as $t_{1} \rightarrow t_{2}$. Hence, we get that $T A$ is equicontinuous. From (41), we have

$$
\begin{aligned}
& \left|\frac{T u(t)}{(1+t)^{\alpha-1}}-\lim _{t \rightarrow \infty} \frac{T u(t)}{(1+t)^{\alpha-1}}\right| \\
& \quad=\left|\frac{T u(t)}{(1+t)^{\alpha-1}}\right| \longrightarrow 0, \quad \text { as } t \longrightarrow \infty .
\end{aligned}
$$

Since

$$
\begin{aligned}
& \left|\int_{t}^{\infty} t a(s) f^{*}\left(s, u(s), D_{0_{+}}^{\alpha-2} u(s), D_{0_{+}}^{\alpha-1} u(s)\right)\right| \\
& \quad \leq \int_{t}^{\infty} \operatorname{ta}(s)\left(H_{0} \phi(s)+1\right) d s \\
& \quad \leq \int_{t}^{\infty} s a(s)\left(H_{0} \phi(s)+1\right) d s \longrightarrow 0, \quad t \longrightarrow \infty, \\
& \lim _{t \rightarrow \infty} D_{0_{+}}^{\alpha-2} T u(t) \\
& \quad=\lim _{t \rightarrow \infty}\left[\int_{0}^{t} s a(s) f^{*}\left(s, u(s), D_{0_{+}}^{\alpha-2} u(s), D_{0_{+}}^{\alpha-1} u(s)\right) d s\right. \\
& \quad+\int_{t}^{\infty} t a(s) f^{*}\left(s, u(s), D_{0_{+}}^{\alpha-2} u(s),\right. \\
& \left.\left.\quad D_{0_{+}}^{\alpha-1} u(s)\right) d s\right] \\
& =\int_{0}^{\infty} s a(s) f^{*}\left(s, u(s), D_{0_{+}}^{\alpha-2} u(s), D_{0_{+}}^{\alpha-1} u(s)\right) d s .
\end{aligned}
$$


which approaches 0 , as $t \rightarrow \infty$. We also have by (45) that

$$
\begin{aligned}
& \left|D_{0_{+}}^{\alpha-1} T u(t)-\lim _{t \rightarrow \infty} D_{0_{+}}^{\alpha-1} T u(t)\right| \\
& \quad=\left|D_{0_{+}}^{\alpha-1} T u(t)\right| \\
& \quad=\int_{t}^{\infty} a(s) f^{*}\left(s, u(s), D_{0_{+}}^{\alpha-1} u(s), D_{0_{+}}^{\alpha-2} u(s)\right) d s,
\end{aligned}
$$

which approaches 0 as $t \rightarrow \infty$. Thus, TA is equiconvergent at $\infty$. Then $T A$ is relatively compact. Therefore, $T$ : $E \rightarrow E$ is completely continuous. The proof is complete.

\section{Main Result}

We are in the position to state the main existence result.

Theorem 14. Let $\gamma, \beta \in E \bigcap L_{\text {loc }}(0, \infty)$ be lower and upper solutions to (5), and suppose that (15) holds. Moreover, $\left(H_{1}\right)-\left(H_{2}\right)$ hold. Then fractional boundary value problem (5) has at least one solution $u(t) \in E \bigcap L_{\mathrm{loc}}(0, \infty)$ satisfying

$$
\begin{gathered}
\gamma(t) \leq u(t) \leq \beta(t), \\
D_{0_{+}}^{\alpha-2} \gamma(t) \leq D_{0_{+}}^{\alpha-2} u(t) \leq D_{0_{+}}^{\alpha-2} \beta(t), \\
\left|D_{0_{+}}^{\alpha-1} u(t)\right| \leq N, \quad \forall t \in[0, \infty),
\end{gathered}
$$

where $N$ is a constant dependent only on $\gamma, \beta$, and $\phi$.

Proof. By Lemma 13, we know that $T: E \rightarrow E$ is completely continuous. By the Schauder fixed point theorem, we can easily obtain that $T$ has at least one fixed point $u \in E$. Thus, $u$ is a solution of (32). Next, we will show that $u$ satisfies the inequalities

$$
\begin{gathered}
\gamma(t) \leq u(t) \leq \beta(t), \\
D_{0_{+}}^{\alpha-2} \gamma(t) \leq D_{0_{+}}^{\alpha-2} u(t) \leq D_{0_{+}}^{\alpha-2} \beta(t), \quad t \in[0, \infty),
\end{gathered}
$$

which implies that $u$ is a solution of (5). First of all, we will show that $D_{0_{+}}^{\alpha-2} u(t) \leq D_{0_{+}}^{\alpha-2} \beta(t)$, for all $t \in[0, \infty)$.

If not, then

$$
\sup _{0 \leq t<\infty}\left(D_{0_{+}}^{\alpha-2} u(t)-D_{0_{+}}^{\alpha-2} \beta(t)\right)>0 .
$$

Note that $\lim _{t \rightarrow \infty}\left(D_{0_{+}}^{\alpha-1} u(t)-D_{0_{+}}^{\alpha-1} \beta(t)\right)<0$; then there are two cases.

Case 1 . There exists a $t_{0} \in[0, \infty)$ such that

$$
\begin{aligned}
& D_{0_{+}}^{\alpha-2} u\left(t_{0}\right)-D_{0_{+}}^{\alpha-2} \beta\left(t_{0}\right) \\
& \quad=\sup _{t \in[0, \infty)}\left(D_{0_{+}}^{\alpha-2} u(t)-D_{0_{+}}^{\alpha-2} \beta(t)\right)>0 .
\end{aligned}
$$

Then $D_{0_{+}}^{\alpha-1} u\left(t_{0}\right)=D_{0_{+}}^{\alpha-1} \beta\left(t_{0}\right)$ and

$$
D_{0_{+}}^{\alpha} u\left(t_{0}\right) \leq D_{0_{+}}^{\alpha} \beta\left(t_{0}\right) .
$$

On the other hand, in view of (32), (33), and $\left(\mathrm{H}_{2}\right)$, we have

$$
\begin{aligned}
& D_{0_{+}}^{\alpha} u\left(t_{0}\right) \\
& =-a\left(t_{0}\right)\left[f\left(t_{0}, w_{0}\left(t_{0}, u\right), w_{1}\left(t_{0}, D_{0_{+}}^{\alpha-2} u\right), D_{0_{+}}^{\alpha-1} u\right)\right. \\
& \left.+\frac{w_{1}\left(t_{0}, D_{0_{+}}^{\alpha-2} u\right)-D_{0_{+}}^{\alpha-2} u\left(t_{0}\right)}{1+\left|w_{1}\left(t_{0}, D_{0_{+}}^{\alpha-2} u\right)-D_{0_{+}}^{\alpha-2} u\left(t_{0}\right)\right|}\right] \\
& =-a\left(t_{0}\right)\left[f \left(t_{0}, w_{0}\left(t_{0}, u\right)\right.\right. \text {, } \\
& \left.w_{1}\left(t_{0}, D_{0_{+}}^{\alpha-2} \beta\left(t_{0}\right)\right), D_{0_{+}}^{\alpha-1} \beta\left(t_{0}\right)\right) \\
& \left.+\frac{D_{0_{+}}^{\alpha-2} \beta\left(t_{0}\right)-D_{0_{+}}^{\alpha-2} u\left(t_{0}\right)}{1+\left|D_{0_{+}}^{\alpha-2} \beta\left(t_{0}\right)-D_{0_{+}}^{\alpha-2} u\left(t_{0}\right)\right|}\right] \\
& \geq-a\left(t_{0}\right) f\left(t_{0}, \beta\left(t_{0}\right), D_{0_{+}}^{\alpha-2} \beta\left(t_{0}\right), D_{0_{+}}^{\alpha-1} \beta\left(t_{0}\right)\right) \\
& +a\left(t_{0}\right) \frac{D_{0_{+}}^{\alpha-2} u\left(t_{0}\right)-D_{0_{+}}^{\alpha-2} \beta\left(t_{0}\right)}{1+\left|D_{0_{+}}^{\alpha-2} u\left(t_{0}\right)-D_{0_{+}}^{\alpha-2} \beta\left(t_{0}\right)\right|} \\
& \geq-a\left(t_{0}\right) f\left(t_{0}, \beta\left(t_{0}\right), D_{0_{+}}^{\alpha-2} \beta\left(t_{0}\right), D_{0_{+}}^{\alpha-1} \beta\left(t_{0}\right)\right) \\
& \geq D_{0_{+}}^{\alpha-1} \beta\left(t_{0}\right)
\end{aligned}
$$

which contradicts (67).

Case 2. Consider $D_{0_{+}}^{\alpha-2} u(0)-D_{0_{+}}^{\alpha-2} \beta(0)=\lim _{t \rightarrow 0^{+}}\left(D_{0_{+}}^{\alpha-2} u(t)-\right.$ $\left.D_{0_{+}}^{\alpha-2} \beta(t)\right)=\sup _{t \in[0, \infty)}\left(D_{0_{+}}^{\alpha-2} u(t)-D_{0_{+}}^{\alpha-2} \beta(t)\right)>0$. By the boundary condition, we have the contradiction $D_{0_{+}}^{\alpha-2} u(0)-$ $D_{0_{+}}^{\alpha-2} \beta(0) \leq 0$. Consequently, $D_{0_{+}}^{\alpha-2} u(t) \leq D_{0_{+}}^{\alpha-2} \beta(t)$ holds for all $t \in[0, \infty)$. By using the similar method, we also can prove that $D_{0_{+}}^{\alpha-2} \gamma(t) \leq D_{0_{+}}^{\alpha-2} u(t)$ for all $t \in[0, \infty)$. By Remark 7, we have

$$
\gamma(t) \leq u(t) \leq \beta(t), \quad \text { for any } t \in[0, \infty)
$$

If $\sigma>0$, we choose

$$
\begin{array}{r}
r \geq \max \left\{\sup _{t \in[\sigma, \infty)} \frac{D_{0_{+}}^{\alpha-2} \beta(t)-D_{0_{+}}^{\alpha-2} \gamma(0)}{t},\right. \\
\left.\sup _{t \in[\sigma, \infty)} \frac{D_{0_{+}}^{\alpha-2} \beta(0)-D_{0_{+}}^{\alpha-2} \gamma(t)}{t}\right\},
\end{array}
$$

and $N>r$ such that

$$
\int_{r}^{N} \frac{s}{h(s)} d s \geq m\left(\sup _{0 \leq t<\infty} D_{0_{+}}^{\alpha-2} \beta(t)-\inf _{0 \leq t<\infty} D_{0_{+}}^{\alpha-2} \gamma(t)\right),
$$

where $m=\sup _{t \in[0, \infty)} a(t) \phi(t)$. From (23), it is clear that $\int_{0}^{\infty} a(s) \phi(s) d s<\infty$. Hence, $m<\infty$. 
Finally, we will check that $\left|D_{0_{+}}^{\alpha-1} u(t)\right|<N$ for $t \in$ $[0, \infty)$. If $\left|D_{0_{+}}^{\alpha-1} u(t)\right| \leq r$, for every $t \in[0, \infty)$, then $\left|D_{0_{+}}^{\alpha-1} u(t)\right|<N$. If $D_{0_{+}}^{\alpha-1} u(t)>r$, for all $t \in[0, \infty)$, then for any $R \geq \sigma$; using (70), we obtain

$$
\begin{aligned}
\frac{D_{0_{+}}^{\alpha-2} \beta(R)-D_{0_{+}}^{\alpha-2} \gamma(0)}{R} & \geq \frac{D_{0_{+}}^{\alpha-2} u(R)-D_{0_{+}}^{\alpha-2} u(0)}{R} \\
& =\frac{\int_{0}^{R}(d / d s)\left(D_{0_{+}}^{\alpha-2} u(s)\right) d s}{R} \\
& =\frac{\int_{0}^{R} D_{0_{+}}^{\alpha-1} u(s) d s}{R} \\
& >r \geq \frac{D_{0_{+}}^{\alpha-2} \beta(R)-D_{0_{+}}^{\alpha-2} \gamma(0)}{R},
\end{aligned}
$$

which is a contradiction. If $D_{0_{+}}^{\alpha-1} u(t)<-r$, for every $t \epsilon$ $[0, \infty)$, we can also have a similar contradiction. There exists $t_{0} \in[0, \infty)$ such that $\left|D_{0_{+}}^{\alpha-2} u\left(t_{0}\right)\right| \leq r$. Thus, there exists $\left[t_{1}, t_{2}\right] \subset[0, \infty)$ such that $\left|D_{0_{+}}^{\alpha-1} u\left(t_{1}\right)\right|=r,\left|D_{0_{+}}^{\alpha-1} u(t)\right|>$ $r, t \in\left(t_{1}, t_{2}\right]$. Otherwise, $\left|D_{0_{+}}^{\alpha-1} u\left(t_{2}\right)\right|=r,\left|D_{0_{+}}^{\alpha-1} u(t)\right|>$ $r, t \in\left[t_{1}, t_{2}\right)$. Without loss of generality, we assume that $D_{0_{+}}^{\alpha-1} u\left(t_{1}\right)=r, D_{0_{+}}^{\alpha-1} u(t)>r, t \in\left(t_{1}, t_{2}\right]$. Therefore, by a convenient change of variable and using (21) and (71), we get

$$
\begin{aligned}
& \int_{D_{0_{+}}^{\alpha-1} u\left(t_{1}\right)}^{D_{0_{+}}^{\alpha-1} u\left(t_{2}\right)} \frac{s}{h(s)} d s \\
& =\int_{t_{1}}^{t_{2}} \frac{D_{0_{+}}^{\alpha-1} u(t)}{h\left(D_{0_{+}}^{\alpha-1} u(t)\right)} D_{0_{+}}^{\alpha} u(t) d t \\
& =\int_{t_{1}}^{t_{2}}-a(t) f\left(t, u(t), D_{0_{+}}^{\alpha-2} u(t), D_{0_{+}}^{\alpha-1} u(t)\right) \\
& \quad \times \frac{D_{0_{+}}^{\alpha-1} u(t)}{h\left(D_{0_{+}}^{\alpha-1} u(t)\right)} d t \\
& \leq \int_{t_{1}}^{t_{2}} a(t) \phi(t) D_{0_{+}}^{\alpha-1} u(t) d t \\
& \leq m \int_{t_{1}}^{t_{2}} D_{0_{+}}^{\alpha-1} u(t) d t \\
& =m\left(D_{0_{+}}^{\alpha-2} u\left(t_{2}\right)-D_{0_{+}}^{\alpha-2} u\left(t_{1}\right)\right) \\
& \leq m\left(\sup _{t \in[0, \infty)} D_{0_{+}}^{\alpha-2} \beta(t)-\inf _{t \in[0, \infty)} D_{0_{+}}^{\alpha-2} \gamma(t)\right) \\
& \leq \int_{r}^{N} \frac{s}{h(s)} d s,
\end{aligned}
$$

which implies that $D_{0_{+}}^{\alpha-1} u\left(t_{2}\right) \leq N$, since $t_{2}$ can be arbitrarily as long as $D_{0_{+}}^{\alpha-1} u(t)>r$; we have $D_{0_{+}}^{\alpha-1} u(t)>r$, for any $t \in[0, \infty)$, which follows that $D_{0_{+}}^{\alpha-1} u(t) \leq N$. By a similar analysis, we can also obtain that if $D_{0_{+}}^{\alpha-1} u\left(t_{1}\right)=-r$, $D_{0_{+}}^{\alpha-1} u(t)<-r, t \in\left(t_{1}, t_{2}\right]$, then $D_{0_{+}}^{\alpha-1} u(t)>-N, t \in[0, \infty)$. Therefore,

$$
\begin{aligned}
D_{0_{+}}^{\alpha} u(t) & =-a(t) f^{*}\left(t, u(t), D_{0_{+}}^{\alpha-2} u(t), D_{0_{+}}^{\alpha-1} u(t)\right) \\
& =-a(t) f\left(t, u(t), D_{0_{+}}^{\alpha-2} u(t), D_{0_{+}}^{\alpha-1} u(t)\right) ;
\end{aligned}
$$

that is, $u$ is a solution of (5), which completes the proof.

Example 15. Consider the boundary value problem of the fractional differential equation on the half-line

$$
\begin{gathered}
D_{0_{+}}^{8 / 3} u(t)+e^{-t}\left(t^{2}+u^{2}(t)\right)\left(\Gamma\left(\frac{5}{3}\right)-D_{0_{+}}^{2 / 3} u(t)\right) \\
\times\left(1+\arctan \left(D_{0_{+}}^{5 / 3} u(t)\right)^{2}\right)=0, \quad t \in(0, \infty), \\
u(0)=D_{0_{+}}^{2 / 3} u(0)=0, \quad D_{0_{+}}^{5 / 3} u(\infty)=0 .
\end{gathered}
$$

In this case, $\alpha=8 / 3, a(t)=e^{-t}, f(t, x, y, z)=\left(t^{2}+\right.$ $\left.x^{2}\right)(\Gamma(5 / 3)-y)\left(1+\arctan \left(z^{2}\right)\right)$. Obviously, $\gamma(t)=-t^{2 / 3}$ and $\beta(t)=t^{2 / 3}$ are a pair of lower and upper solutions of (75). Furthermore, we have $\gamma, \beta \in E, \gamma(t) \leq \beta(t)$, for $t \in[0, \infty)$. It is clear that $f$ is continuous on $[0, \infty) \times \mathbb{R}^{3}$. If $0 \leq t<\infty$, $-t^{2 / 3} \leq x \leq t^{2 / 3},-\Gamma(5 / 3) \leq y \leq \Gamma(5 / 3)$, we have

$$
|f(t, x, y, z)| \leq \phi(t) h(|z|),
$$

where $\phi(t)=4 \Gamma(5 / 3)\left(1+t^{2}\right)$ and $h(z)=1+z^{2}$; we have

$$
\int_{0}^{\infty} \frac{s}{h(s)} d s=\int_{0}^{\infty} \frac{s}{1+s^{2}} d s=\infty,
$$

and $f$ satisfies the like Nagumo condition with respect to $-t^{2 / 3}, t^{2 / 3}$. Furthermore, we have

$$
\begin{gathered}
\int_{0}^{\infty} \max \{s, 1\} a(s) d s=\int_{0}^{1} e^{-s} d s+\int_{1}^{\infty} s e^{-s} d s<\infty, \\
\int_{0}^{\infty} \max \{s, 1\} a(s) \phi(s) d s \\
=4 \Gamma\left(\frac{5}{3}\right) \int_{0}^{1}\left(1+s^{2}\right) e^{-s} d s \\
+4 \Gamma\left(\frac{5}{3}\right) \int_{1}^{\infty} s\left(1+s^{2}\right) e^{-s} d s<\infty .
\end{gathered}
$$

Thus, we conclude by Theorem 14 that there exists at least one solution $u(t)$ to boundary value problem (75) such that

$$
\begin{aligned}
-t^{2 / 3} & \leq u(t) \leq t^{2 / 3}, \\
-\Gamma\left(\frac{5}{3}\right) \leq D_{0_{+}}^{\alpha-2} u(t) & \leq \Gamma\left(\frac{5}{3}\right), \quad t \in[0, \infty) .
\end{aligned}
$$

\section{Conflict of Interests}

The authors declare that there is no conflict of interests regarding the publication of this paper. 


\section{Acknowledgments}

The authors thank the referees for their valuable and helpful suggestions and comments that improved the paper. This work is supported by the Natural Science Foundation of Jiangsu Province (BK2011407) and the Natural Science Foundation of China (11271364 and 10771212).

\section{References}

[1] R. P. Agrwal and D. O'Regan, Infinite Interval Problems for Differential, Difference and Integral Equations, Kluwer Academic Publisher, Dodrecht, The Netherlands, 2001.

[2] R. P. Agrwal and D. O’Regan, “Infinite interval problems modeling phenomena which arise in the theory of plasma and electrical potential theory," Studies in Applied Mathematics, vol. 111, no. 3, pp. 339-358, 2003.

[3] J. V. Baxley, "Existence and uniqueness for nonlinear boundary value problems on infinite intervals," Journal of Mathematical Analysis and Applications, vol. 147, no. 1, pp. 122-133, 1990.

[4] C. Bai and J. Fang, "On positive solutions of boundary value problems for second-order functional differential equations on inifinite intervals," Journal of Mathematical Analysis and Applications, vol. 282, no. 2, pp. 711-731, 2003.

[5] C. Bai and C. Li, "Unbounded upper and lower solution method for third-order boundary-value problems on the half-line," Electronic Journal of Differential Equations, vol. 2009, no. 119, pp. $1-12,2009$.

[6] H. Lian and F. Geng, "Multiple unbounded solutions for a boundary value problem on infinite intervals," Boundary Value Problems, vol. 2011, no. 51, pp. 1-8, 2011.

[7] S. Liang and J. Zhang, "The existence of countably many positive solutions for some nonlinear three-point boundary problems on the half-line," Nonlinear Analysis, Theory, Methods and Applications, vol. 70, no. 9, pp. 3127-3139, 2009.

[8] Y. Liu, "Existence and unboundedness of positive solutions for singular boundary value problems on half-line," Applied Mathematics and Computation, vol. 144, no. 2-3, pp. 543-556, 2003.

[9] B. Liu, L. Liu, and Y. Wu, "Unbounded solutions for three-point boundary value problems with nonlinear boundary conditions on $[0,+\infty), "$ Nonlinear Analysis, Theory, Methods and Applications, vol. 73, no. 9, pp. 2923-2932, 2010.

[10] J. Li, B. Liu, and L. Liu, "Solutions for a boundary value problem at resonance on $[0, \infty)$, " Mathematical and Computer Modelling, vol. 58, no. 11-12, pp. 1769-1776, 2013.

[11] H. Lian and W. Ge, "Solvability for second-order three-point boundary value problems on a half-line," Applied Mathematics Letters, vol. 19, no. 10, pp. 1000-1006, 2006.

[12] R. P. Agarwal, V. Lakshmikantham, and J. J. Nieto, "On the concept of solution for fractional differential equations with uncertainty," Nonlinear Analysis, Theory, Methods and Applications, vol. 72, no. 6, pp. 2859-2862, 2009.

[13] B. Ahmad and J. J. Nieto, "Existence results for a coupled system of nonlinear fractional differential equations with threepoint boundary conditions," Computers and Mathematics with Applications, vol. 58, no. 9, pp. 1838-1843, 2009.

[14] C. Bai, "Impulsive periodic boundary value problems for fractional differential equation involving Riemann-Liouville sequential fractional derivative," Journal of Mathematical Analysis and Applications, vol. 384, no. 2, pp. 211-231, 2011.
[15] C. Bai, "Existence result for boundary value problem of nonlinear impulsive fractional differential equation at resonance," Journal of Applied Mathematics and Computing, vol. 39, no. 1-2, pp. 421-443, 2012.

[16] K. Diethelm and N. J. Ford, "Analysis of fractional differential equations," Journal of Mathematical Analysis and Applications, vol. 265, no. 2, pp. 229-248, 2002.

[17] I. Podlubny, Fractional Differential Equations, in: Mathematics in Sciences and Engineering, vol. 198, Academic Press, San Diego, Calif, USA, 1999.

[18] S. G. Samko, A. A. Kilbas, and O. I. Marichev, Fractional Integrals and Derivatives. Theory and Applications, Gordon and Breach, Yverdon, Switzerland, 1993.

[19] V. E. Tarasov, Fractional Dynamics: Applications of Fractional Calculus to Dynamics of Particles, Fields and Media,, Springer, New York, NY, USA, 2011.

[20] Y. Chen and X. Tang, "Positive solutions of fractional differential equations at resonance on the half-line," Boundary Value Problems, vol. 2012, no. 64, pp. 1-13, 2012.

[21] C. Kou, H. Zhou, and Y. Yan, "Existence of solutions of initial value problems for nonlinear fractional differential equations on the half-axis," Nonlinear Analysis, Theory, Methods and Applications, vol. 74, no. 17, pp. 5975-5986, 2011.

[22] X. Su and S. Zhang, "Unbounded solutions to a boundary value problem of fractional order on the half-line," Computers and Mathematics with Applications, vol. 61, no. 4, pp. 1079-1087, 2011.

[23] A. Arara, M. Benchohra, N. Hamidi, and J. J. Nieto, "Fractional order differential equations on an unbounded domain," Nonlinear Analysis, Theory, Methods and Applications, vol. 72, no. 2, pp. 580-586, 2009.

[24] D. Baleanu, O. G. Mustafa, and D. O’Regan, "On a fractional differential equation with infinitely many solutions," Advances in Difference Equations, vol. 2012, no. 145, pp. 1-6, 2012.

[25] X. Liu and M. Jia, "Multiple solutions of nonlocal boundary value problems for fractional differential equations on the halfline," Electronic Journal of Qualitative Theory of Differential Equations, vol. 2011, no. 56, pp. 1-14, 2011.

[26] S. Liang and J. Zhang, "Existence of three positive solutions of $\mathrm{m}$-point boundary value problems for some nonlinear fractional differential equations on an infinite interval," Computers and Mathematics with Applications, vol. 61, no. 11, pp. 33433354, 2011.

[27] Y. Lin, B. Ahmad, and R. P. Agarwal, "Existence of solutions for a coupled system of nonlinear fractional differential equations with fractional boundary conditions on the half-line," Advances in Difference Equations, vol. 2013, no. 46, pp. 1-19, 2013.

[28] X. Su, "Solutions to boundary value problem of fractional order on unbounded domains in a Banach space," Nonlinear Analysis, Theory, Methods and Applications, vol. 74, no. 8, pp. 2844-2852, 2011.

[29] G. Wang, B. Ahmad, and L. Zhang, "A coupled system of nonlinear fractional differential equations with multipoint fractional boundary conditions on an unbounded domain," Abstract and Applied Analysis, vol. 2012, Article ID 248709, 11 pages, 2012.

[30] L. Zhang, G. Wang, B. Ahmad, and R. P. Agarwal, "Nonlinear fractional integro-differential equations on unbounded domains in a Banach space," Journal of Computational and Applied Mathematics, vol. 249, pp. 51-56, 2013.

[31] X. Zhao and W. Ge, "Unbounded solutions for a fractional boundary value problems on the infinite interval," Acta Applicandae Mathematicae, vol. 109, no. 2, pp. 495-505, 2010. 
[32] D. Delbosco and L. Rodino, "Existence and uniqueness for a nonlinear fractional differential equation," Journal of Mathematical Analysis and Applications, vol. 204, no. 2, pp. 609-625, 1996.

[33] M. Meehan and D. O'Regan, "Existence theory for nonlinear Fredholm and Volterra integral equations on half-open intervals," Nonlinear Analysis, Theory, Methods and Applications, vol. 35, no. 3, pp. 355-387, 1999.

[34] B. Yan, D. O'Regan, and R. P. Agarwal, "Unbounded solutions for singular boundary value problems on the semi-infinite interval: upper and lower solutions and multiplicity," Journal of Computational and Applied Mathematics, vol. 197, no. 2, pp.365386, 2006. 


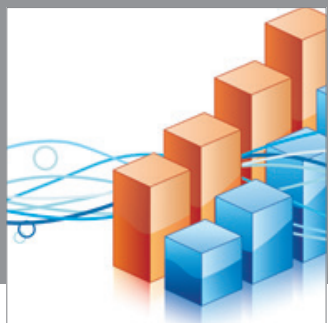

Advances in

Operations Research

mansans

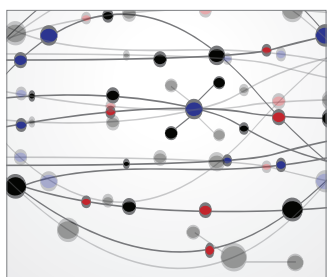

The Scientific World Journal
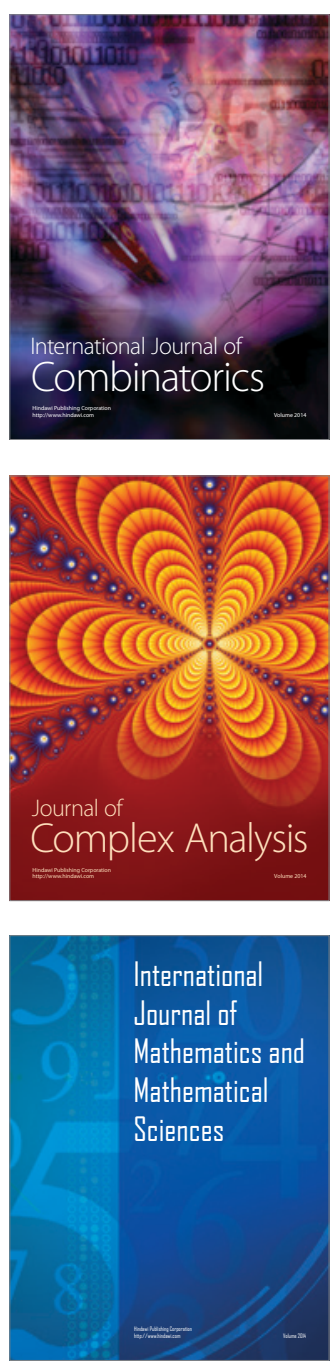
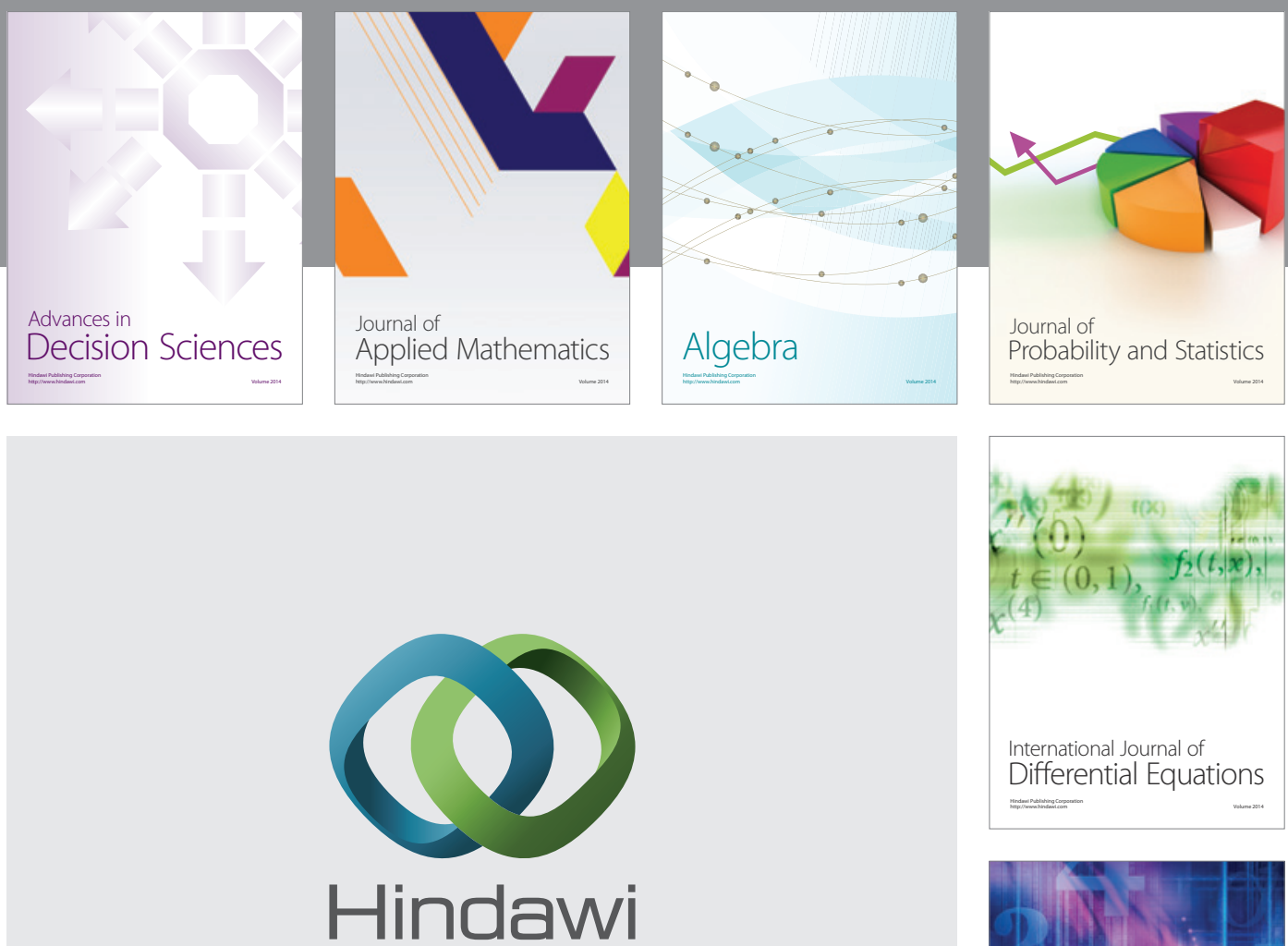

Submit your manuscripts at http://www.hindawi.com
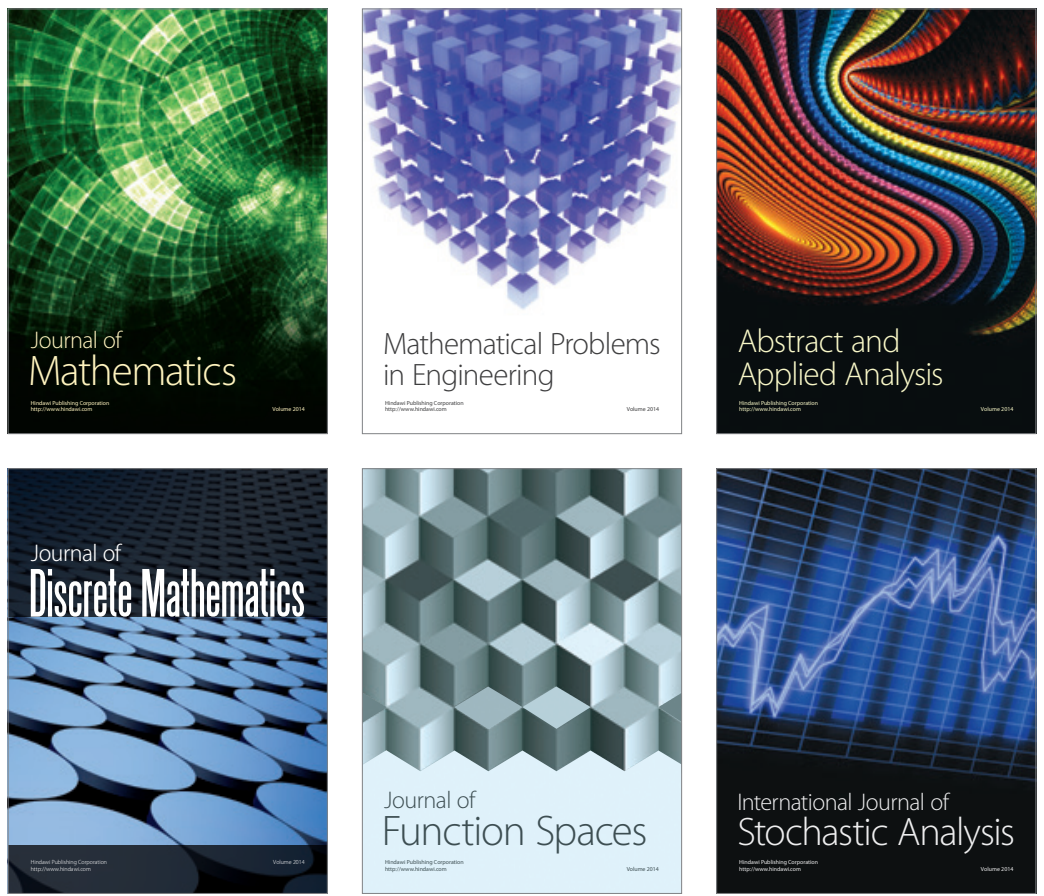

Journal of

Function Spaces

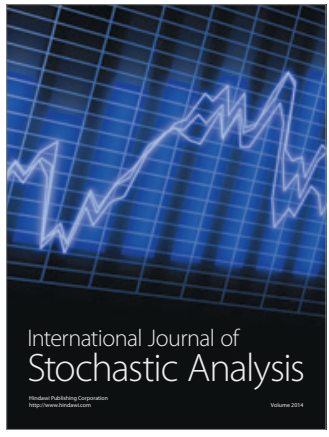

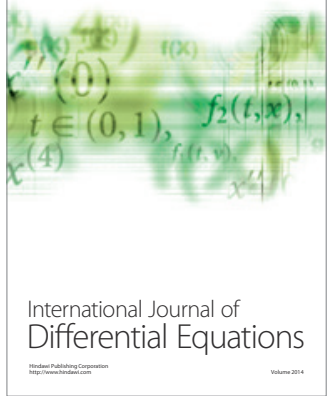
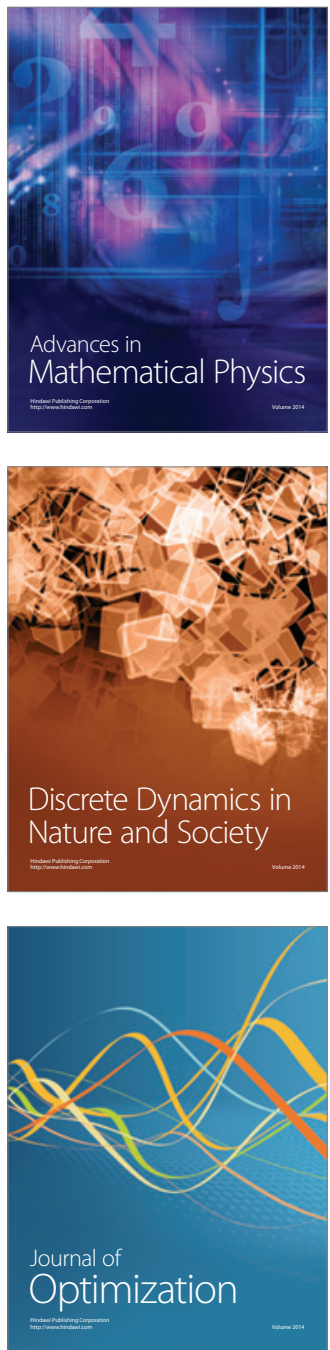